\title{
Core-Shell Type Polystyrene Latex Possessing Reactive Poly(ethylene glycol) Brushes on the Surface for High Performance Immunodiagnostics
}

\author{
Ryutaro OGawa, Yukio Nagasaki, ${ }^{\dagger}$ Naoya ShIBATA, ${ }^{*}$ Hidenori OTsuKa, ${ }^{* *}$ \\ and Kazunori KATAOKA*** \\ Department of Materials Science and Technology, Tokyo University of Science, 2641 Yamazaki, \\ Noda, Chiba 278-8510, Japan \\ ${ }^{*}$ Research and Development NanoCarrier Co., Ltd., Tokatsu Techno Plaza, 5-4-6 Kashiwanoha, \\ Kashiwa, Chiba 277-0882, Japan \\ **Artificial Organ Materials Research Group, Biomaterials Center, National Institute of Materials Science, 1-1 \\ Namiki, Tsukuba 305-0044, Japan \\ ${ }^{* * *}$ Department of Materials Science, Graduate School of Engineering, The University of Tokyo, \\ 7-3-1 Hongo, Bunkyo-ku, Tokyo 113-8656, Japan
}

(Received June 14, 2002; Accepted October 16, 2002)

\begin{abstract}
Amphiphilic poly(ethylene glycol)-b-polylactide (PEG/PLA) block macromonomers with an aldehyde group at the PEG chain end and a methacryloyl group at the PLA chain end were quantitatively synthesized by anionic polymerization. PEGylated latex particles were then prepared by dispersion radical copolymerization of the PEG/PLA block macromonomer with styrene in water. PEG/PLA macromonomer itself works as a surfactant to stabilize styrene droplets in the copolymerization process. Acetal groups at the PEG chain end on the latex particle were quantitatively converted to aldehyde groups by an acid treatment. In this way, polystyrene latex particles covered with PEG tethered chains possessing an aldehyde end-group were obtained. The size of the latex was quantitatively controlled ranging in size from $56 \mathrm{~nm}$ to $235 \mathrm{~nm}$ by the amount of the block macromonomer. The number of aldehyde groups on the latex particle was estimated to be approximately 0.029 molecules $\mathrm{nm}^{-2}$ by electric spin resonance (ESR) using 4-amino2,2,6,6-tetramethylpiperidine-1-oxyl (TEMPO) as a probe. Regardless of the size of the latex particles synthesized in this study, the occupied area of 4-amino-TEMPO on the particle was constant. Biotin was then introduced in a distal end of PEG-strands as a model ligand. The biotin-modified PEGylated latex was coagulated in a facile manner by the addition of avidin. From an inhibitory coagulation test using free biotin, it was found that more than $5.8 \times 10^{8}$ times of free biotin was required for the prevention of coagulation. Such a high complex formation ability of the biotin on the latex surface to avidin may be explained by the hypervalency effect. The core-shell type polystyrene latex particles, with reactive PEG brushes thus prepared, show a stable dispersity and a lowered non-specific interaction with proteinous compounds, and may have a promising potential in the diagnostic field.

KEY WORDS Polystyrene Latex / Heterobifunctional Block Macromonomer / Aldehyde Group / Ractive Poly(ethylene glycol) Brush / Immunodiagnostic /
\end{abstract}

Latex particles ranging in size from submicron to a few hundred micrometers have been extensively utilized in versatile fields such as paints, inks, adhesives and rubber. The method for controlling size and the size distribution of latex particles has been well established. ${ }^{1}$ In 1956, Singer and Plotz reported that polystyrene latex particles adsorbed with immuno gamma globulin $\mathrm{G}$ ( $\operatorname{IgG}$ ) can be utilized in immuno diagnostics, ${ }^{2}$ because the immuno-coagulation response was enhanced by the use of an IgG adsorbed latex particle. Since then, such a latex-based immunodiagnostic system has been widely utilized and is known as a turbidimetric assay method.

When polystyrene-based latex is used in the immunodiagnostic field, non-specific adsorption of protein and cellular components to the latex surface is one of the major obstacles because of its hydrophobic propen-

${ }^{\dagger}$ To whom correspondence should be addressed. sity. Ito et al. reported that dispersion polymerization of styrene in the presence of poly(ethylene glycol) macromonomers gave polystyrene latex possessing PEG tethered chains on the surface. ${ }^{3}$ This type of approaches reduces the non-specific adsorption of biocomponents due to the decreased interfacial free energy as well as increased steric repulsion of the tethered PEG chains on the surface. On the other hand, there is no functionality to introduce immuno ligands on the surface of the PEG-latex thus prepared even though the stability of the particle was significantly improved. Akashi et al. recently reported the preparation of polystyrene latex possessing poly(methacrylic acid) tethered chains on the surface by the copolymerization of styrene with the poly(tert-butyl methacrylate) macromonomer followed by acid hydrolysis. The flanking carboxylic acid groups of the tethered chains can be utilized for the immobilization of ligand 
molecules.

Recently, we reported a facile and quantitative synthesis of a heterobifunctional $\mathrm{PEG},{ }^{4-8}$ which denotes a PEG having a functional group at one end and another functional group at the other end. By using the heterobifunctional PEG as a macroinitiator, new heterobifunctional $\mathrm{AB}$ block copolymers were also synthesized, retaining the discriminate functionality at both chain ends. One of such a heterotelechelic block copolymers, acetal-PEG/poly(lactide)OCOC $\left(\mathrm{CH}_{3}\right)=\mathrm{CH}_{2}$, possesses several functionalities; hydrophilic-hydrophobic amphiphilic character lends it a property nonionic surfactant to stabilize styrene droplets in the aqueous dispersion polymerization. The methacryloyl end group can be incorporated into the latex via covalent linkage to form stable PEG tethered chains on the surface. The acetal end group can then be converted to an aldehyde group that can be utilized for further bio-conjugation. This paper deals with the study on the dispersion polymerization of styrene in the presence of acetal-PEG/PLA-OCOC $\left(\mathrm{CH}_{3}\right)=\mathrm{CH}_{2}$ for preparation of functionalized PEG-polystyrene latex. The characterizations of the surface functionality as well as the application to protein-recognition assay are also described.

\section{EXPERIMENTAL SECTION}

\section{Materials}

Commercial tetrahydrofuran (THF), 3,3-diethoxypropanol (Aldrich), 2-methoxyethanol (WAKO), DL-lactide (LA) (Aldrich), methacrylic anhydride (Aldrich), styrene (Kanto Chemical) and 2,2'-azobisisobutyronitrile (AIBN) (WAKO) were purified by conventional methods. ${ }^{9}$ Ethylene oxide (EO) (Sumitomo Seika Chemicals) was dried over calcium hydride and distilled under argon atmosphere. Potassium naphthalene was used as a THF solution, whose concentration was determined by titration. Other reagents were used as received.

\section{Synthesis of Heterobifunctional Block Macromonomer} ( $\alpha$-Acetal- $\omega$-methacryloyl-PEG/PLA)

$\alpha$-Acetal- $\omega$-methacryloyl-PEG/PLA block macromonomers (acetal-PEG/PLA-MA) were synthesized by a one-pot anionic ring-opening polymerization of EO followed by LA using potassium 3,3diethoxypropanolate (PDP) as an initiator at room temperature under argon. $2.0 \mathrm{mmol}(0.315 \mathrm{~mL})$ of 3,3diethoxypropanol and $2.0 \mathrm{mmol}$ of potassium naphthalene were added to $60 \mathrm{~mL}$ of dry THF to form PDP. After the mixture was stirred for several minutes, appropriate amounts of condensed EO were added via a cooled syringe to the formed PDP solution. The polymerization of the EO proceeded for $2 \mathrm{~d}$ at room temperature. $6.94 \mathrm{~mL}$ of LA solution in THF $(c=$ $1.00 \mathrm{~mol} \mathrm{~L}^{-1}$ ) was introduced to the mixture, and then, the reaction was continued for $120 \mathrm{~min}$. After the polymerization reaction, $10 \mathrm{mmol}(1.49 \mathrm{~mL})$ of methacrylic anhydride was further added into the polymer solution, and the reaction mixture was stirred for $2 \mathrm{~d}$. The polymer was recovered by precipitation into a 20 -fold excess of cold isopropyl alcohol $\left(-15^{\circ} \mathrm{C}\right)$, and centrifuged for $30 \mathrm{~min}$ at $5000 \mathrm{rpm}(4723 \times \mathrm{g})$ at $-4^{\circ} \mathrm{C}$. The recovered polymer was freeze-dried from benzene. The yield of the obtained polymer was $c a .90 \%$.

\section{Polymer Characterization}

The molecular weight of the PEG segment was determined by gel permeation chromatography (GPC) at the end of the EO polymerization. The molecular weight of the PLA segment was determined by the ${ }^{1} \mathrm{H}$ NMR spectrum by the ratio of methine protons in the PLA segment $v s$. methylene protons in the PEG segment based on the number-average molecular weight $\left(M_{\mathrm{n}}\right)$ of PEG determined from the GPC result. GPC measurements were carried out using a TOSOH HLC8120 equipped with a TSK gel columns (TSKgel SuperHZ4000 + HZ3000 + HZ2500) and an internal refractive index (RI) detector (TOSOH HLC-8020RI). THF containing $0.5 \mathrm{wt} \%$ triethylamine was used as the eluent at a flow rate of $0.35 \mathrm{~mL} \mathrm{~min}{ }^{-1}$ at $40{ }^{\circ} \mathrm{C}$. The ${ }^{1} \mathrm{H}$ NMR spectrum was obtained using chloroform- $d$ solutions with a JEOL EX400 spectrometer at $400 \mathrm{MHz}$.

\section{Preparation of Latex Particles}

One of the representative procedures is described here: $0.4 \mathrm{mmol}$ of acetal-PEG/PLA-MA (PEG/PLA = $4,500 / 280)$ and $0.438 \mathrm{mmol}$ of AIBN were dissolved in $43.6 \mathrm{mmol}(4.11 \mathrm{~mL})$ of styrene. The mixture was dispersed in $150 \mathrm{~mL}$ of degassed water in a $500 \mathrm{~mL}$ round-bottomed flask equipped with a mechanical rotator. The mixture was heated at $60^{\circ} \mathrm{C}$ for $24 \mathrm{~h}$ with stirring at $400 \mathrm{rpm}$. After the polymerization, a solid mass of polystyrene was removed by filtration. The reaction mixture was dialyzed against water for several days to remove the unreacted styrene. The conversion of the surface acetal groups into aldehyde groups was conducted directly after the dialysis. The latex particle solution was adjusted to $\mathrm{pH} 2.0$ with hydrochloric acid and stirred for $2 \mathrm{~h}$ at room temperature. After the $\mathrm{pH}$ of the mixture was neutralized with $\mathrm{NaOH}(\mathrm{aq})$, the solution was dialyzed against water to remove the salt. 


\section{Characterization of Latex Particles}

The size and polydispersity of latex particles were determined from field emission scanning electron micrograph (FE-SEM, HITACHI S-4200B) images. Cumulant diameter of the latex particles was determined by dynamic light scattering (DLS) (DLS-7000 Photal, Otsuka Electronics equipped with a $18 \mathrm{~mW}$ Ar ion laser that produces vertically polarized incident beams at $\lambda_{0}=488 \mathrm{~nm}$ ).

\section{Modification of the PEG Chain End on the Latex Sur- face}

In order to evaluate the number of aldehyde groups on the latex surface, electron spin resonance (ESR) measurements were carried out after modification of the aldehyde groups by 4-amino-TEMPO. The latex particle solution was centrifuged at $20000 \mathrm{rpm}(75576 \times \mathrm{g})$ and dispersed into borate buffer $(50 \mathrm{mM}, \mathrm{pH} 8.0)$ so as to adjust the concentration of the latex particle solution to $20.0 \mathrm{mg} \mathrm{mL}^{-1}$. Borate buffer containing $1.82 \times 10^{-2} \mathrm{~mol} \mathrm{~L}^{-1}$ of 4-amino-TEMPO (Aldrich) was added into $2 \mathrm{~mL}$ of the latex particle borate buffer solution. After the mixture was stirred for $30 \mathrm{~min}$, $0.38 \mathrm{mmol}$ of sodium cyanoborohydride $\left(\mathrm{NaBH}_{3} \mathrm{CN}\right)$ was added for reductive amination. The reaction mixture was stirred for $24 \mathrm{~h}$ at room temperature; then, the mixture was dialyzed against borate buffer for $24 \mathrm{~h}$. The electron spin intensity was monitored by an ESR spectrometer (JEOL JES-RE2X) using $\mathrm{Mn}^{2+}$ as the standard signal, operating at the X-band frequency of $9.15 \mathrm{GHz}$, scanning from 3190 to $3340 \mathrm{G}$.

\section{Biotin-modified Latex Particles}

$2.00 \mathrm{~mL}$ of $1.05 \mathrm{mmol} \mathrm{L}^{-1}$ biocytin hydrazide (PIERCE) aqueous solution and $2.00 \mathrm{~mL}$ of $1.00 \mathrm{mg} \mathrm{mL}^{-1}$ latex particle solution were mixed in a $15-\mathrm{mL}$ flask, and reacted for $5 \mathrm{~h}$. The reaction mixture was dialyzed against water for $8 \mathrm{~h}$ to remove unreacted biocytin hydrazide.

\section{Latex Agglutination Test}

$0.5 \mathrm{~mL}$ of $0.5 \mathrm{mg} \mathrm{mL}^{-1}$ biotinylated latex particle solution was added to various concentrations of avidin (Sigma), lysozyme (Sigma) or bovine serum albumin (Sigma) aqueous solution. Absorbance at $340 \mathrm{~nm}$ was monitored using a Shimadzu UV-2400PC 300 s after mixing.

After the latex agglutination test was carried out by the use of avidin (fluorescein conjugate) (Molecular Probes), the imaging of the luminescence was taken by a fluorescent microscope using a Nanofinder system 110 (Tokyo Instruments).

\section{Inhibition of Latex Agglutination}

The biotinylated latex particle solution in the presence of various concentrations of free biocytin hydrazide was added to $0.5 \mathrm{~mL}$ of $0.01 \mathrm{mg} \mathrm{mL}^{-1}$ avidin aqueous solution. Absorbance at $340 \mathrm{~nm}$ was monitored using a Shimadzu UV-2400PC $300 \mathrm{~s}$ after mixing.

\section{RESULTS AND DISCUSSION}

\section{Synthesis of $\alpha$-Acetal- $\omega$-methacryloyl-PEG/PLA Block Macromonomers}

The synthesis of PEG macromonomers as well as the dispersion polymerization using these PEG monomers has so far been extensively reported. ${ }^{10-16}$ PEG macromonomers possessing a hydrophobic spacer in between the PEG chain and the polymerizable double bond was prepared as a monomer with an appreciable surfactant ability for dispersion polymerization. Because most of the PEG macromonomers prepared so far possess no functional group at the distal chain end, the prepared latex lacks functionality on the surface. To solve this problem, we synthesized amphiphilic PEG/PLA heterobifunctional block macromonomers with an $\alpha$-formyl PEG and hydrophobic PLA of varying chain length. For this purpose, one-pot anionic ring-opening polymerization of EO followed by LA was performed using a functional initiator. An aldehyde end group was chosen as a functional group because it is stable in aqueous media, and is easy to react with an amine under reducing conditions. The initiator with an aldehyde group, however, cannot be used for the anionic polymerization of EO, because of the active hydrogen in the aldehyde group. Therefore, we employed a potassium alcoholate possessing an acetal group as an initiator for EO polymerization because the acetal group can be easily converted to the aldehyde group by acid treatment. For $\omega$-terminal functionalization, methacrylic anhydride was used as an electrophilic agent, and was added to terminate the polymerization. The molecular weight of both the PEG and PLA segments can be controlled by changing the initial monomer/initiator ratio, resulting in the preparation of PEG/PLA block macromonomer samples with varying composition as summarized in Table I. The molecular weight of the PEG segment was determined by GPC at the end of the EO polymerization. The profile of subsequent block copolymerizations was monitored by GPC at the end of the reaction, indicating the unimodal and narrow molecular weight distribution of block macromonomers used in this study. The $M_{\mathrm{w}}$ of the obtained PEG was in good accordance with the initial monomer/initiator ratio $\left([\mathrm{EO}]_{0} /[\mathrm{PDP}]_{0}\right)$. Because an adsorption of PEG/PLA block copolymer onto gel in 
Table I. Characterization of PEG-PLA Blockmacromonomers

\begin{tabular}{lccccc}
\hline & \multicolumn{3}{c}{ PEG } & PLA \\
\cline { 2 - 4 } & $M_{\mathrm{n}}(\mathrm{GPC})$ & $M_{\mathrm{w}}(\mathrm{GPC})$ & $M_{\mathrm{w}} / M_{\mathrm{n}}(\mathrm{GPC})$ & & $M_{\mathrm{n}}$ (NMR) \\
\hline Blockmacromonomer Type 1 & 6900 & 7450 & 1.08 & & 5000 \\
Blockmacromonomer Type 2 & 4500 & 4800 & 1.07 & & 280 \\
\hline
\end{tabular}

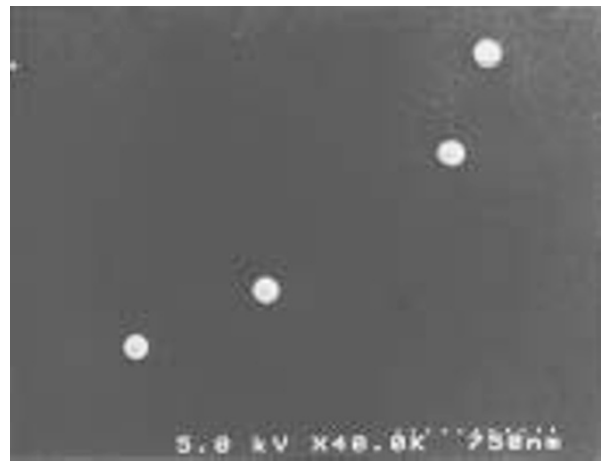

Figure 1. SEM photograph of typical polystyrene latex particles (latex 7).

the GPC analysis tends to occur, the molecular weight of the PLA segment was determined using the ${ }^{1} \mathrm{H}$ NMR spectrum ${ }^{17}$ based on the $M_{\mathrm{n}}$ of the PEG segment determined from the GPC results.

\section{Preparation of Polystyrene Latex Possessing Reactive- PEG Tethered Chains on the Surface}

Polystyrene latex particles were prepared by dispersion polymerization in the presence of heterobifunctional block macromonomers. The solubilized macromonomer works not only as a comonomer in the polymerization but also as a surfactant. Figure 1 shows an FE-SEM photograph of the obtained latex particles. The obtained latexes showed spherical structure and their size distribution was very narrow. Using the data obtained from the SEM photograph, the size and its distribution factor were determined as $129 \mathrm{~nm}$ and $1.01(\mathrm{dw} / \mathrm{dn})$, respectively. Assuming a complete spherical structure of the latex, a histogram analysis was carried out by the DLS measurement as shown in Figure 2. The size distribution data shown in Figure 2, again confirmed the unimodal distribution of the latex. The average diameter and its distribution determined by a cumulant method were $131.4 \mathrm{~nm}$ and 0.03 $\left(\mu \Gamma^{-2}\right)$, respectively, agreeing well with the SEM data. The data obtained under different polymerization conditions are summarized in Table II. With increasing the block macromonomer concentration, the size of the obtained latex decreased as anticipated (Figure 3). Jan reported that the surface activity of block copolymers obtained by the addition of ethylene oxide to $n$-hexyl, $n$-octyl, and $n$-decyl ethers of mono- to tetrabutylene glycol in aqueous solutions increases with the length of the alkyl group and with the number of oxybutylene



Figure 2. Gamma distribution of the polystyrene latex particle (latex 7) analyzed by DLS.

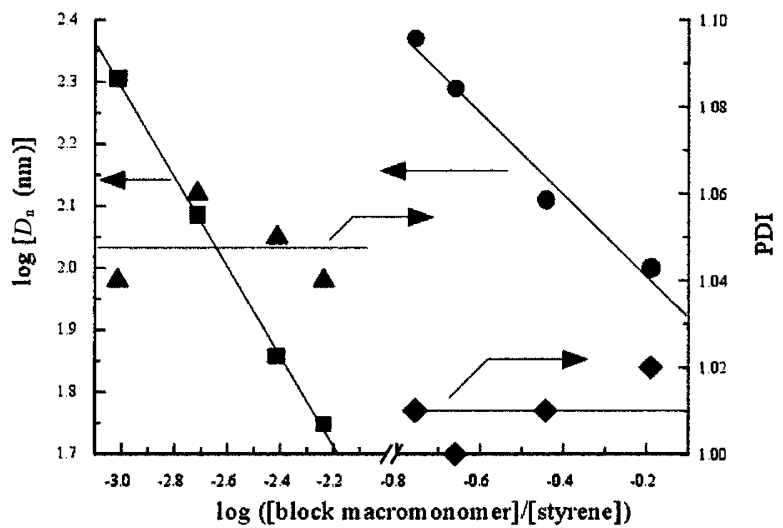

Figure 3. Double-logarithmic plot of number average diameter $\left(D_{\mathrm{n}}\right)$ of latex particle $v s$. [blockmacromonomer]/[styrene] and polydispersity index (PDI) of latex particle $v s$. [blockmacromonomer]/ [styrene]. $D_{\mathrm{n}}$ of latex particle synthesized using macromonomer Type $1(\mathbb{\square})$, Type $2(\bullet)$. PDI of latex particle synthesized using macromonomer Type $1(\boldsymbol{\Delta})$, Type $2(\bullet)$.

groups. ${ }^{18}$ The acetal-PEG/PLA-MA having a large $M_{\mathrm{w}}$ of the PLA segment (Type 1) revealed higher surfactant ability than that having a short PLA segment (Type 2). The size distribution of the latex obtained from a Type 1 macromonomer, however, was wider than that from a Type 2 macromonomer. Presumably, immiscibility of the PLA segment with polystyrene may influence the broader size distribution of the former system. On the basis of these results, we concluded that the size of the latex was controlled by the [macromonomer]/[styrene] ratio. Notably, the macromonomer having a shorter PLA chain (Type 2) gave the latex with extremely low size distribution $(\leq 1.02)$.

The stability of the latexes prepared in this study was fairly stable. For example, no precipitation was observed during several months storage in refrigerator $\left(4{ }^{\circ} \mathrm{C}\right)$. 
Table II. The recipe of preparing latex paticle, the number average diameter and polydispersity index (PDI)

\begin{tabular}{ccccccc}
\hline & PEG-PDLLA $(\mathrm{g})$ & $\mathrm{St}(\mathrm{mL})$ & AIBN $(\mathrm{mg})$ & Water $(\mathrm{mL})$ & $\begin{array}{c}\text { Number average } \\
\text { diameter }(\mathrm{nm})\end{array}$ & PDI \\
\hline latex 1 & $0.05^{\mathrm{a}}$ & 0.50 & 7.20 & 15.0 & 202 & 1.04 \\
latex 2 & $0.10^{\mathrm{a}}$ & 0.50 & 7.20 & 15.0 & 122 & 1.06 \\
latex 3 & $0.20^{\mathrm{a}}$ & 0.50 & 7.20 & 15.0 & 72.0 & 1.05 \\
latex 4 & $0.30^{\mathrm{a}}$ & 0.50 & 7.20 & 15.0 & 56.0 & 1.04 \\
latex 5 & $1.97^{\mathrm{b}}$ & 27.0 & 386 & 400 & 235 & 1.01 \\
latex 6 & $2.97^{\mathrm{c}}$ & 27.0 & 387 & 400 & 194 & 1.00 \\
latex 7 & $4.89^{\mathrm{c}}$ & 27.0 & 387 & 400 & 129 & 1.01 \\
latex 8 & $8.59^{\mathrm{d}}$ & 27.0 & 388 & 400 & 100 & 1.02 \\
\hline \multicolumn{5}{c}{${ }^{\mathrm{a}}$ PEG/PDLLA $=6900 / 5000}$. & ${ }^{\mathrm{b}}$ PEG/PDLLA $=4500 / 280$. & ${ }^{\mathrm{c}}$ PEG/PDLLA $=5500 / 250$. \\
dPEG/PDLLA $=5500 / 140$. &
\end{tabular}

(a)

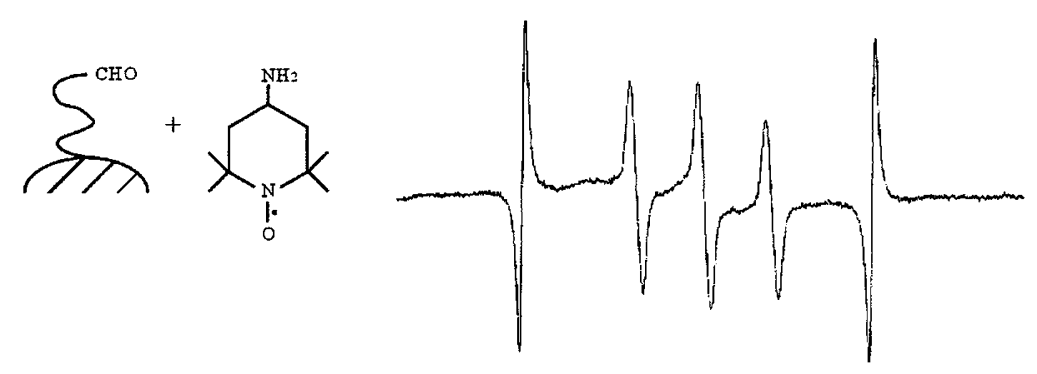

(b)
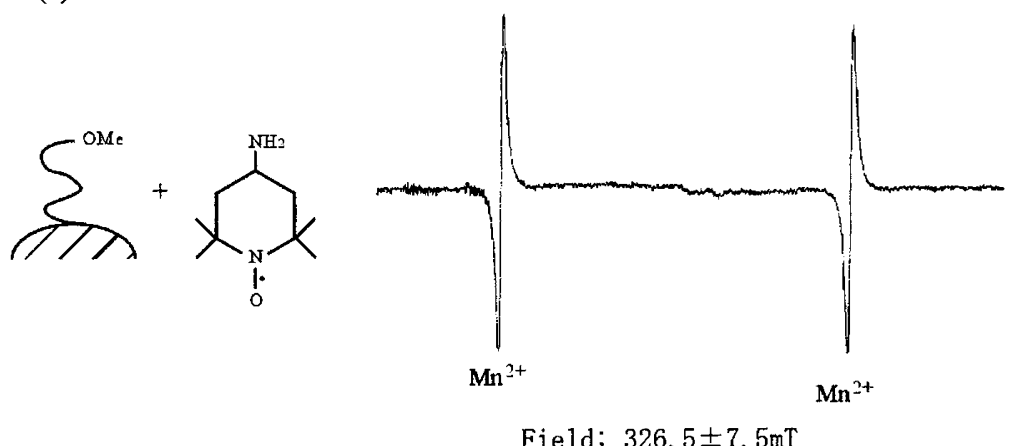

Figure 4. ESR spectra after the reaction between the latex particle and 4-amino-TEMPO: (a) aldehyde latex particle - 4-amino-TEMPO; (b) Methoxy latex particle - 4-amino-TEMPO systems.

\section{ESR Study of the Aldehyde-PEG-Latex}

The conversion of the acetal end into aldehyde was conducted by acid hydrolysis at $\mathrm{pH} 2$ with hydrochloric acid for $5 \mathrm{~h}$. After the hydrolysis reaction, the latex solution was neutralized and dialyzed against water. The quantity of aldehyde groups on the latex particle was determined by an ESR probe method. Since the aldehyde group reacts rapidly with a primary amino group under reducing condition, 4-amino-TEMPO was introduced to the PEG end as the ESR probe. Sodium cyanoborohydride $\left(\mathrm{NaBH}_{3} \mathrm{CN}\right)$ was chosen as the reducing agent. As shown in Figure 4a, clear triplet signals were observed after the latex was treated with 4-amino-TEMPO. On the contrary, no signal was observed when the same experiment was carried out using the PEG-latex without aldehyde groups (Figure 4b).
These results indicate the occurrence of the effective covalent conjugation of 4-amino-TEMPO with the aldehyde group at the end of PEG on the latex surface. Quantitative analysis of the TEMPO-radical of the PEG-latex was carried out using several samples with different particle size. Assuming the density of the latex to be $1.0 \mathrm{~g} \mathrm{~cm}^{-3}$ with a spherical shape, the number of TEMPO on the latex surface was calculated as summarized in Figure 5. Apparently, with increasing size of the particle, the number of TEMPO molecules on the surface was increased.

Wu et al. reported that the average surface area occupied by each PEG molecule is a constant regardless of the size of the latex obtained in the presence of PEG macromonomers, ${ }^{19}$ which is in good agreement with that of our data shown in Figure 5. They also re- 


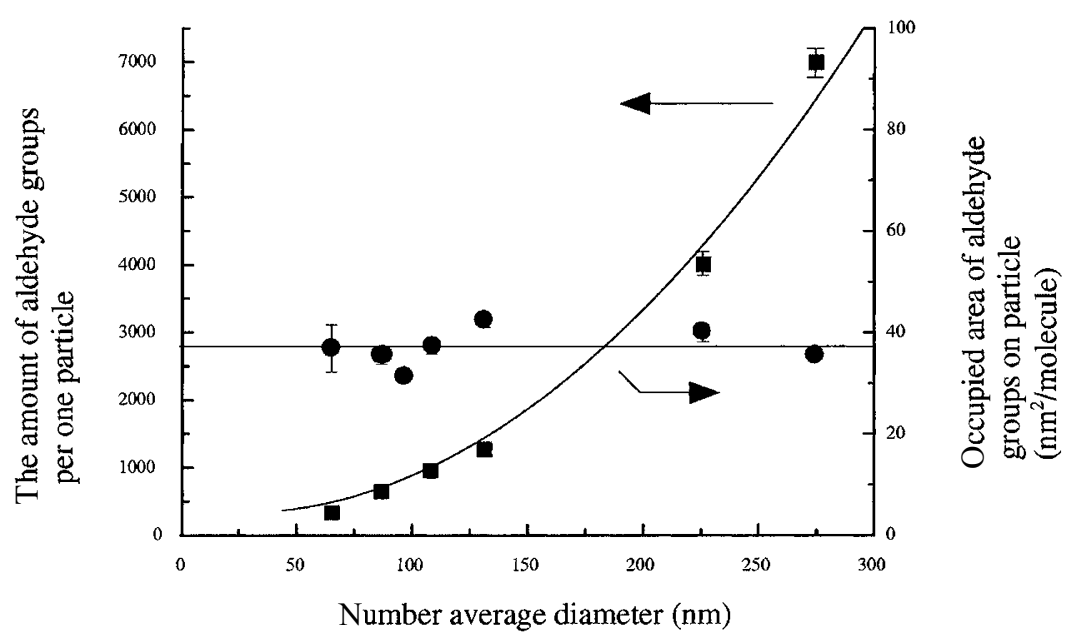

Figure 5. The amount of aldehyde groups per one particle and the value of the occupied area of aldehyde groups on particle.

ported that the surface area should be $1 \mathrm{~nm}^{2}$ providing that all of the macromonomers locate on the surface of the latexes. Nevertheless, the calculation based on the data obtained by our ESR measurements is $35 \mathrm{~nm}^{2}$. Considering the high reactivity of the aldehyde group to the primary-amine under reducing conditions, most of the PEG free-end should be converted to the ESR probe. This means that only $3 \%$ of PEG chain ends locates on our latex surface compared to Wu's calculation. This may be reasonable because the copolymerization of monomer with macromonomer starts from the initial stage of the polymerization reaction in the droplet. During the polymerization, the small droplets coagulate to grow latex. Thus, a certain amount of PEG macromonomer should be incorporated into the core of the latex. Actually, our data were close to the experimental results $\left(25 \mathrm{~nm}^{2}\right)$ reported by Wu et al. ${ }^{19}$

\section{Molecular Recognition by Biotinylated PEG-Latex}

As stated above, the polystyrene latex possessing aldehyde PEG tethered chains on the surface was prepared under a controlled manner. The surface with the PEG tethered chains is well known to avoid nonspecific adsorption of biocomponents such as proteins and cells. Thus, the obtained latex is anticipated to avoid non-specific adsorption. The aldehyde group at the PEG chain end can be further modified with a specific ligand used for immunodiagnostics with the advantage of suppressing non-specific interactions. In this study, biotin was used as a model ligand because of its high specificity to avidin protein. ${ }^{20}$

It is well known that hydrazine reacts easily with an aldehyde group without any reducing agent to form a stable Schiff base. Thus, biotin possessing a watersoluble hydrazine linkage (biocytin hydrazide) was utilized for the biotinylation of the aldehyde-PEG latex. The reaction was carried out by just adding a 3-fold of biocytin hydrazide against the aldehyde group to the aldehyde-PEG latex suspension in aqueous medium. After the dialysis of the suspension, the biotinylated PEG latex was utilized for molecular recognition experiments.

When the biotinylated-PEG-latex $(157 \mathrm{~nm})$ was mixed with BSA, no change in turbidity was observed, regardless of the amount of BSA concentration (Figure 6). Lysozyme gave the same phenomena as BSA. On the contrary, significant change in the turbidity of the solution was observed when avidin was mixed with the biotinylated-PEG-latex. The turbidity change was monitored in detail as a function of avidin concentration $v s$. the biotin on the surface of the latex. In the region of lower avidin concentration ([avidin]/[biotin] $<0.5$ ), the solution was transparent due to the excess biotin-latex molecules on the latex surface. With increasing avidin concentration to exceed [avidin] $/[$ biotin $]=0.5$, the solution became turbid. The turbidity became maximum when [avidin] $/[$ biotin] $=$ 2 , and then, decreased. In this maximized region in turbidity, an effective crosslinking occurred between avidin and biotinylated-PEG-latex. In the region of [avidin]/[biotin] $>10$, no more turbidity change was observed, which is explained by the excess avidin molecules $v s$. the biotin on the latex surface.

These phenomena were monitored by fluorescent microscope using fluorescein-conjugated avidin. The fluorescence was observed in the region where turbidity emerges, while isolated latexes were observed in the region of [avidin]/[biotin] $>10$, indicating that avidin covered the latex surface.

In order to confirm the specific molecular recognition between the avidin-biotin couple, biotin-inhibitory tests were carried out. When free biotin was added to the biotinylated-PEG-latex mixed with avidin, a competitive interaction between free biotin and biotinylated- 


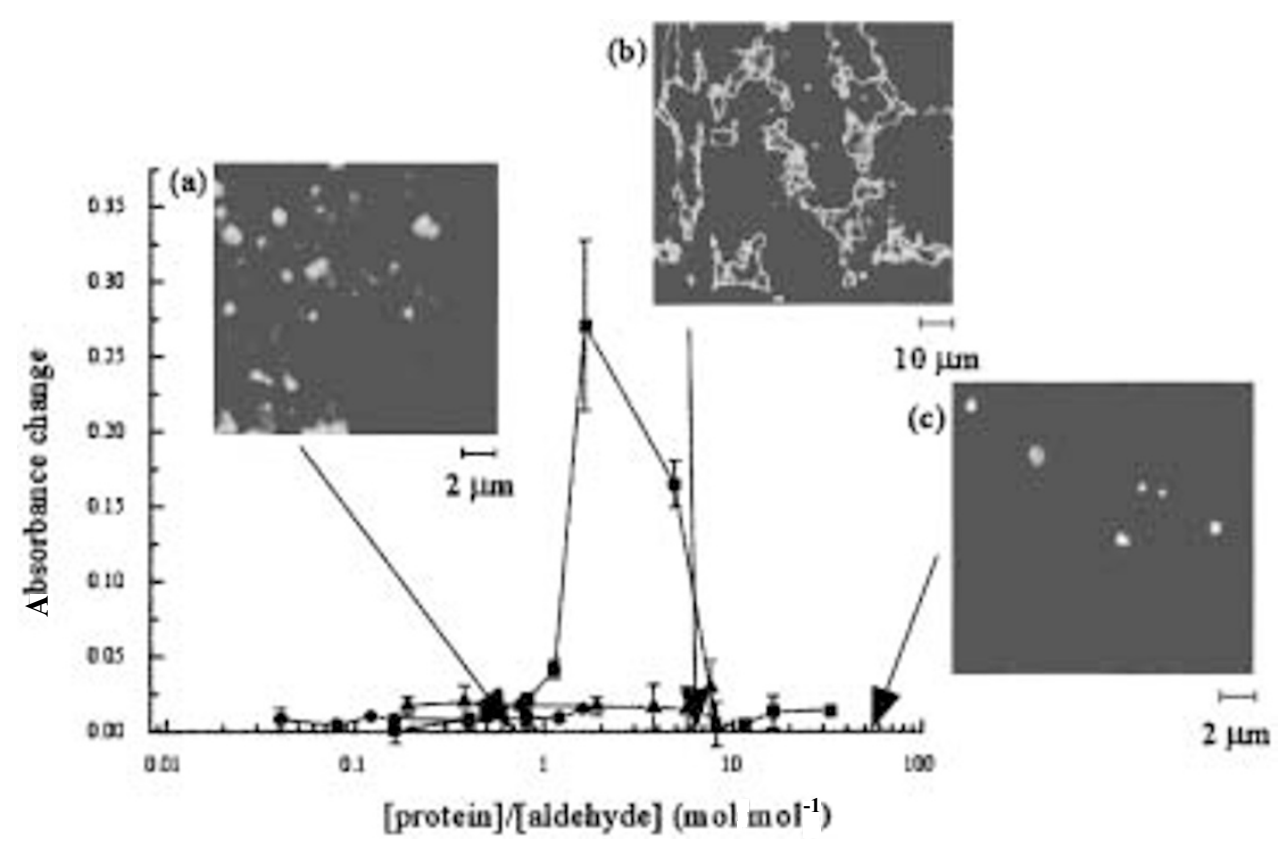

Figure 6. Latex agglutination test; ( $\mathbf{\square})$ avidin, ( $)$ BSA, ( $(\mathbf{\Delta})$ lysozyme. Images (a), (b), and (c) are Nanofinder images by the use of avidin (fluorescein conjugate). The agglutination of latex particle could be detected by absorbance at $340 \mathrm{~nm}$ using UV-vis spectrometer. Biocytin hydrazide modified latex particle solution was mixed with avidin solution in quartz cell. After $300 \mathrm{~s}$, the value of absorbance was read. Latex particle which diameter was $157.3 \mathrm{~nm}$ (measured by DLS) was used in this experiment and concentration of latex particles in react solution was $0.25 \mathrm{mg} \mathrm{mL}^{-1}$.

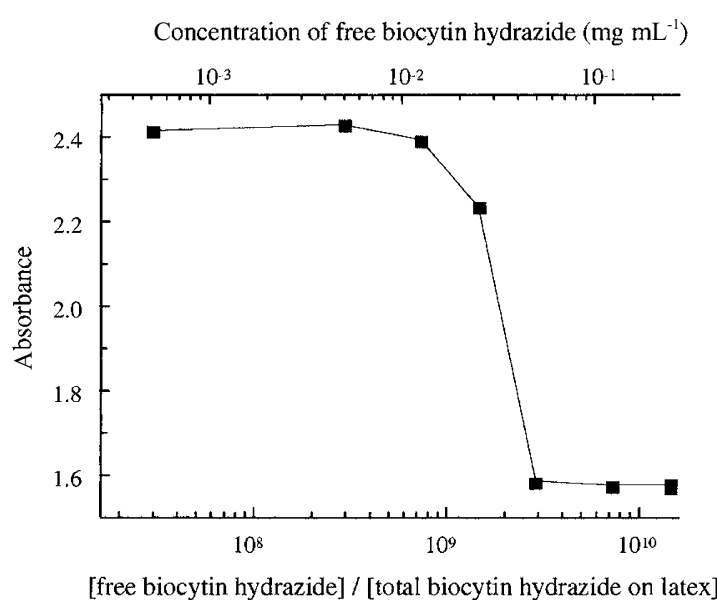

Figure 7. Inhibition of latex agglutination. Biocytin hydrazide modified latex particle solution was mixed with avidin solution in the presence of free biocytin hydrazide. The concentration of avidin in reaction mixture was $0.005 \mathrm{mg} \mathrm{mL}^{-1}$, which was max absorbance change point in Figure 6.

latex should take place. Figure 7 shows change in the turbidity as a function of free biotin concentration in the biotin-latex solution coupled with avidin. When the free biotin concentration was less than $1 \times 10^{-2} \mathrm{mg} \mathrm{mL}^{-1}$, which denotes $5.8 \times 10^{8}$ times higher concentration than biotins immobilized on the latex surface, no turbidity change was observed. More than $10^{9}$ times higher concentration of free biotin than avidin was required to inhibit the interaction of avidin with the latex. Whitesides et al. reported that hypervalency interaction plays a substantial role in the case of ligands carrying by a high polymer matrix. ${ }^{21}$ If ligands directly locate on a solid surface, however, the affinity with the receptor should decrease significantly, which reduces performance of several systems such as affinity chromatography and immobilized enzymes.

In the present latex system, the ligand locates on the free end of PEG chain tethered on the latex surface, meaning that the PEG chains work as flexible spacers. We have already revealed that the mobility of the free end at the PEG tethered chain increased with increasing molecular weight of the PEG. ${ }^{22}$ At a PEG molecular weight of more than 5000, ligand mobility at the free chain end became the same order as that in the solution. Since the latex prepared in this study possesses PEG chains with a $M_{\mathrm{w}}$ of $c a$. 6900 , the mobility of the ligand biotin at the PEG chain end should be almost the same as that in solution. Along with the hypervalency effect, the high mobility of ligand may contribute to the facile contact of biotin to avidin in the solution. This may be the reason for such a high value of free biotin inhibition to avidin by latex-immobilized biotin molecules.

\section{CONCLUSION}

Heterobifunctional block macromonomers, $\alpha$-acetal$\omega$-methacryloyl-PEG/PLA, were synthesized and used as a functional surface active reagent. Dispersion polymerization of polystyrene latex particles was carried out by using heterobifunctional block macromonomers, 
followed by the conversion of the acetal group into an aldehyde group in acidic aqueous solution. The number average diameter $\left(D_{\mathrm{n}}\right)$ of latex particles was dependent on the [block macromonomer]/[styrene] ratio. The number of aldehyde groups on a latex particle was determined by an ESR probe method. It was clear that the value of the occupied area of aldehyde groups on a particle is about $35 \mathrm{~nm}^{2}$. The aldehyde groups on the surface were converted to biotin molecule using biocytin hydrazide. Biotinylated latex thus prepared was effectively coagulated with avidin addition. Inhibitory tests using free biotin to the biotin-latex-avidin system showed higher apparent affinity of latex-immobilized biotin, which can be explained by the integrated effect of flexible PEG spacers and hypervalency.

\section{REFERENCES}

1. E. S. Daniels, E. D. Sudol, and M. S. El-Aasser, Ed., "Polymer Latexes", American Chemical Society, Washington, D.C., 1992.

2. J. M. Singer and C. M. Plotz, Am. J. Med., 21, 888 (1956).

3. S. Kawaguchi, M. A. Winnik, and K. Ito, Macromolecules, 28, 1159 (1995).

4. Y. J. Kim, Y. Nagasaki, K. Kataoka, M. Kato, M. Yokoyama, T. Okano, and Y. Sakurai, Polym. Bull., 33, 1 (1994).

5. S. Cammas, Y. Nagasaki, and K. Kataoka, Bioconjugate Chem., 6, 226 (1995).

6. Y. Nagasaki, T. Kutsuna, M. Iijima, M. Kato, and K. Kataoka,
Bioconjugate Chem., 6, 231 (1995).

7. Y. Nagasaki, M. Iijima, M. Kato, and K. Kataoka, Bioconjugate Chem., 6, 702 (1995).

8. Y. Nagasaki, R. Ogawa, S. Yamamoto, M. Kato, and K. Kataoka, Macromolecules, 30, 6489 (1997).

9. D. D. Perrin, W. L. F., F. W. Moor, Ed., "Purification of Laboratory Chemicals", 2nd ed, Pergamom Press, Oxford, 1980.

10. M. Maniruzzaman, S. Kawaguchi, and K. Ito, Macromolecules, 33, 1583 (2000).

11. M. Akashi, D. Chao, E. Yashima, and N. Miyauchi, J. Appl. Polym. Sci., 39, 2027 (1990).

12. J. Liu, C. H. Chew, and L. M. Gan, J. macromol. Sci., Pure Appl. Chem., A33, 337 (1996).

13. Y. Wang and J. Huang, Macromolecules, 31, 13 (1998).

14. J. S. Shay, R. J. English, R. J. Spontak, C. M. Balik, and S. A. Khan, Macromolecules, 33, 6664 (2000).

15. A. Brindley, S. S. Davis, M. C. Davies, and J. F. Watts, J. Colloid Interface Sci., 171, 150 (1995).

16. I. Capec, "Macromolecular Reports", A32(Suppls. 5\&6), 1995, pp 749-758.

17. Y. Nagasaki, T. Okada, C. Scholz, M. Iijima, M. Kato, and K. Kataoka, Macromolecules, 31, 1473 (1998).

18. J. Chlebicki, J. Collod Interface Sci., 206, 77 (1998).

19. C. Wu, M. Akashi, and M.-Q. Chen, Macromolecules, 30, 2187 (1997).

20. M. Wilchek and E. A. Bayer, Ed., "Methods in Enzymology, Volume 184", Academic Press, Inc., New York, N.Y., 1990.

21. M. Mammen, S.-K. Choi, and G. M. Whitesides, Angew. Chem. Int. Ed., 37, 2754 (1998).

22. H. Otsuka, Y. Nagasaki, and K. Kataoka, Biomacromolecules, 1, 39 (2000). 